\title{
INTERCULTURAL ASPECTS OF ONLINE COMMUNICATION A COMPARISON OF MANDARIN-SPEAKING, US, EGYPTIAN AND GERMAN USER PREFERENCES
}

\author{
Margarete Seidenspinner ${ }^{1}$, Gabriele Theuner ${ }^{2}$ \\ Heilbronn University, Max-Planck-Str. 39, 73081 Heilbronn, Germany, \\ Ludwigshafen University of Applied Sciences, Ernst-Böhe-Str. 4, 67059 Ludwigshafen am Rhein \\ E-mails: ${ }^{1}$ seidenspinner@hs-heilbronn.de, ${ }^{2}$ theuner@fh-ludwigshafen.de
}

Received 10 November 2006; accepted 3 February 2007

\begin{abstract}
This paper sums up the results of a survey conducted with four samples of website users, i.e. young urban academics from the PR of China, the United States, Egypt and Germany. The survey analyses the ways in which users from such distinctly dissimilar cultural environments view websites. The websites researched are those of global operators that either offer international services, high price utility items or technologically advanced products.

The results gained in the course of this research project demonstrate how the targeted users' cultural environments may impact on their preferred navigation tools, their perceived quality of web designs and on the perception and processing of the information provided online. A brief overview of the consequences which derive from this culture-bound user behaviour for corporate communication policies conclude this paper.
\end{abstract}

Keywords: culture-bound user preferences, China, Egypt, US, Germany, impact of culture on website ranking, cultural adaptation of websites, young urban academic website users.

\section{Introduction}

Global business organisations have increasingly used the internet to expand and develop their e-commerce ventures and to communicate information about their products and services to and in the Middle and the Far East. As online presence is closely linked to PR goals and sales targets, web performance has become a crucial area of corporate communication policies. This also results from the fact that online communication subscribing to ethnocentric standards has not proved to be as effective as anticipated. This applies particularly to target groups originating from cultural environments that differ fundamentally from those of the product or service providers.

Businesses that serve a range of culturally diverse markets have tried to meet the challenges of the IT era by resorting to a large variety of proven quantitative and qualitative approaches to optimise their online communications. They have employed a plethora of conventional layout and style guidelines which, nonetheless, have often failed to give due consideration to the cultural aspects of online designs with regard to the user groups they target.

\section{Rationale, goals and objectives}

The research endeavour described in the following was designed to explore and identify the needs, preferences and behavioural patterns of young urban internet users as precisely as possible and to establish the similarities and differences in the way in which they perceive, recognize and process the verbal and pictorial website information they are viewing.

It was assumed that this behaviour would markedly differ according to certain specific aspects inherent in the users' cultural environment.

Why have cultural issues grown to such importance in corporate life? Ethnographic consumer research has provided ample proof of the fact that the recognition and - above all - the processing of visual information depends largely on the degree of the involvement of the users and that the degree of this involvement 
is highly influenced by individual value ${ }^{1}$ systems. The level of involvement influences buying decisions, for instance, and encourages or discourages repeat purchases and product recommendations. These value systems are the result of education, emulation (e.g. of peer groups or parental example) and the socialisation process that each individual goes through and determine his or her cultural affinities. However, "culture" is an intangible phenomenon that can only be comprehended by its observable facets and products (e.g. artefacts, thoughts, speech, behaviour).

For heuristic reasons and for the purpose of this paper, "culture" will be seen as a knowledge pool shared by the members of a specific in-group (here: the four samples) that informs this group's behaviour. Cultural expressions such as corporate websites are immediately recognisable to in-group members but may represent a hermetic system to outsiders [1-3].

The objectives of this research project are directly related to this, namely:

- to identify the most relevant web tools for young academics and the website features they prefer;

- to explore the cultural aspects which influence the perception and information processing of this group of users;

- to point out what consequences this entails for web design and for corporate marketing communication policies.

\section{Research method and literature review}

The epistemological research approach of this project derives from the objectives specified above. It relies on the collection of empirical materials by electronic observation, interviews and questionnaires. SPSS software was then used to analyse the primary data. In order to gain a deeper insight into the research subject at hand and to be able to induct a grounded theory from it, a series of interpretive ethnographic practices were included, such as first-person accounts, and ethnographic reports and case studies, which directly bear on the research proposition $[4,5]$.

Ethnographic research of this nature relies on participant observation, whose role requires both detachment and personal involvement. The risks involved

1 Values systems consist of a great number of inter-related attitudes. Some attitudes hold a focal position thereby exerting an influence on a large number of behaviours and consequently affect entire areas of a person's life. These central attitudes are defined as values: in other words, "values" represent attitudes that are particularly sound and resist change. in this have long been documented. On the one hand, direct emphatic involvement of the researcher may lead to a loss of precision in research detail, on the other hand, it allows direct access to specific user behaviour within the highly complex ICT context [6].

A further snag involved in "participant observation" is the possible ethnocentricity of the researchers that may prevent them from asking the most appropriate questions and may induce them to produce causal attributions which impede the interpretation of survey results [6]. To overcome this, the samples were integrated into the evaluation of the results and the recommendations made. Moreover, region-specific publications were used to provide insider an insight into the cultural issues relevant to this study.

The project detailed below relies to a large extent on verbal survey techniques, which track and document intuitive judgements such as the ones made on the effect of the advertising sites and on the nature of the users' own perception. This method assumes that the statements made by the respondents can be taken at face value and that the underlying attitudes can be reliably described. The reliability - or the potential lack thereof - of such first-person narratives has also been widely discussed.

Notwithstanding these reservations, the empirical data collected allows a preliminary analysis of the website usage of the samples at hand.

In order to bind the respondents more closely to this work, semi-structured interviews were conducted after the results had been analysed in the course of which the respondents were questioned about their personal value systems which are decisive in the generation and the perception of information.

\section{The Survey}

\subsection{Type and structure of the samples}

Four samples were used in this survey whose structure was largely dictated by pragmatic aspects such as the availability of respondents and the accessibility of background information.

Sample 1: The first part of the survey was conducted with 28 students from the PR of China (19 to 31 years old) who had recently come to Germany to improve their language skills and to prepare for a broad range of university courses at the Goethe-Institute in Mannheim.

Sample 2: The second sample included 64 senior business students (18 to 28 years old) enrolled at a private university in Florence, South Carolina. 
Sample 3: This group consisted of 22 Egyptian exchange students (17 to 21 years old) from Misr International University, a private institution of Higher Education located in Cairo, who had come to Germany as exchange students. They came from diverse academic backgrounds, such as business, communications, pharmacy and architecture.

Sample 4: 56 German students (19 to 31 years) from the two universities participating in this survey. They were divided into two groups to match the Chinese sample $(n=34)$ and the Egyptian one $(n=22)$.

The four samples are comparable in terms of age, education, family background and, above all, internet experience. All respondents stated that they frequently surfed the net and used online services (Fig 1).

Moreover, the large number of students contributing to this survey possess a significant level of intercultural awareness or have had some relevant intercultural experience. They take an active interest in cultural issues and are aiming at employment in internationally operating companies.

In spite of the cultural and linguistic diversity of the respondents, they formed a relatively homogeneous group of internet users (young, middle-class academics, internationally-minded, mobile and internet experienced) that can be directly targeted if preferences and group-specific behaviours are taken account of.

\subsection{Choosing the websites}

The websites crucial to this survey differ essentially in layout, contents, style and communication goals. They were chosen

- for their different degrees of standardisation and adaptation to the local markets, but, above all;

- for their assumed affective appeal to the samples;

- they all advertise valued consumer items and services associated with technological innovation, adventure and leisure.

The first set of websites advertises the services of two national airlines that are also used by sample members. They are intended to attract a broad range of domestic and international customers to and from their home base.

China Eastern is a Shanghai-based subsidiary of the national carrier Air China. The company uses online formats whose screen layouts, contents, navigation tools and colours are completely standardized but whose languages are entirely adapted to their domestic and international markets (Japan, South Korea, India, the UK and Australia). Two versions of the website were available: one in Mandarin and one in English [7-9].

The website's central cover-all feature is the website script offering details of the company's shooting star history, the PR of China's economic clout, the "commercial capital" of its home base Shanghai and China Eastern's major destinations. "Hospitality" and "affordability" are mentioned as essential customer values. Contrary to Western airlines, the company also suggests reasons for perceived weaknesses (i.e. slow international updating) in order to forestall customer complaints [7-9]. Services, customer advice and other links are placed in a column on the left hand side where determined users look for flight details and booking services (Fig 1).

The second website type features the Mercedes-Benz models which represent a global brand. DaimlerChrysler use a hybrid communication format which mirrors the corporation's "globalisation" strategy [7]. Exhibit 2 shows that the company seeks to combine the sophisticated lifestyle they associate with their cars (beautiful natural environment, luxury and wealth, open spaces, high-brow vocabulary) with typical local landscape elements (Fig 2).

The central or slightly off-centre position of the cars attracts the viewers' immediate attention. The language of the advertising message is factual but erudite. It presents the S-type as a symbol of opulence, cuttingedge technology and supreme service quality. The site is clearly aimed at the new class of wealthy business people that wish to underline their status by buying a luxury car model.

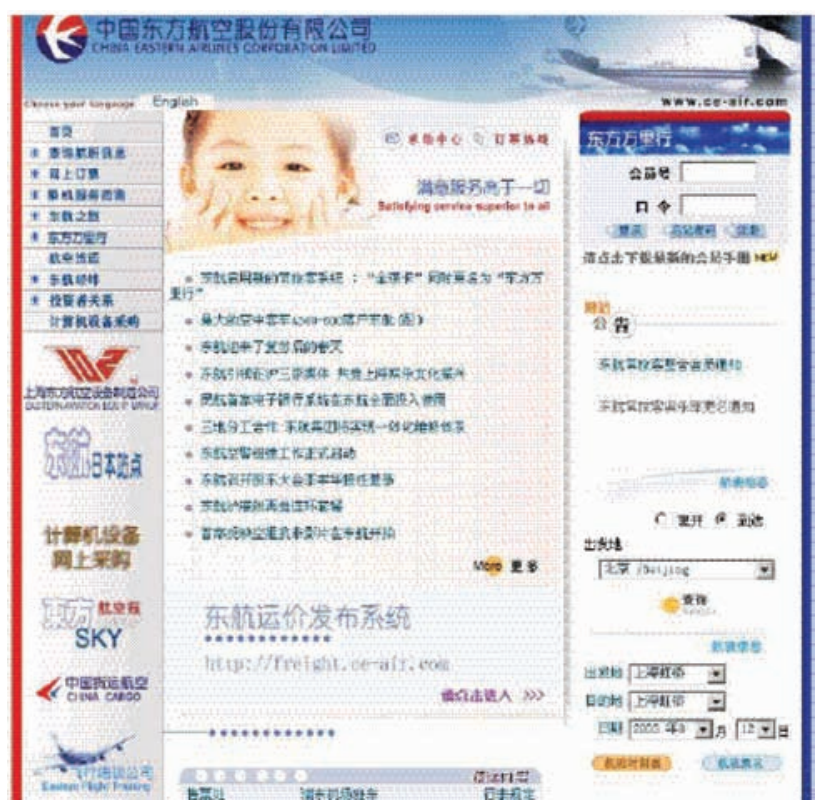

Fig 1. China Eastern targeting their domestic market 


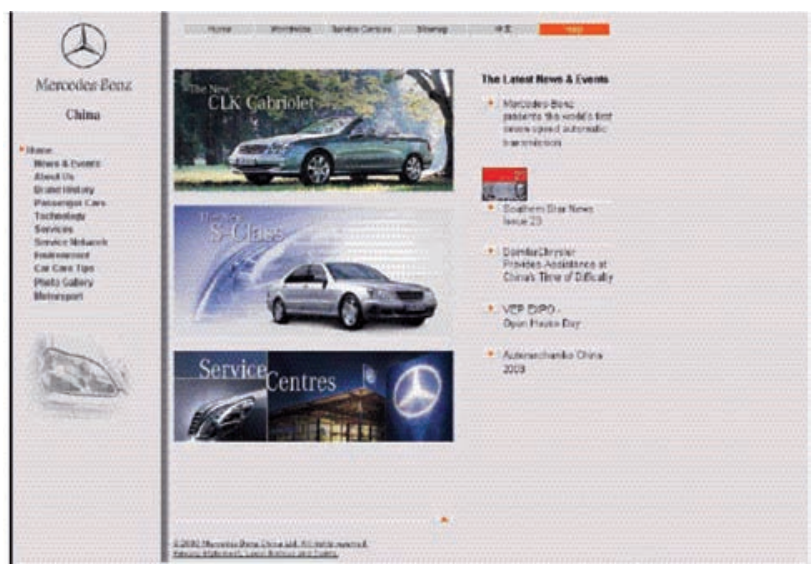

Fig 2. Mercedes-Benz China: Selling an opulent lifestyle

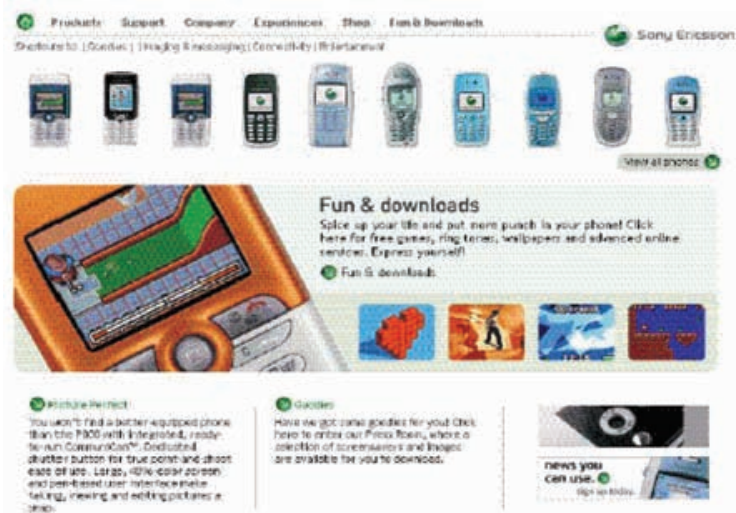

Fig 3. Sony Ericsson's US website

The third variety of websites used was Sony Ericsson's online presentation of its most recent generation of mobile phones. This company uses animated portals whose latest version remains approximately identical in all its markets. The websites are either fully or partially translated into the languages spoken in the targeted markets. They emphasize the large variety of models available and their newly developed electronic features (Fig 3). The language is streetwise and aimed at young consumers, the purchase promise is that of fun.

\subsection{Collecting the data}

The survey proceeded through four stages. During the pre-test stage, relevant demographic data of the sample was collected including age and gender. Moreover, the users were requested to specify their level of internet experience which was generally felt to be high.
This was followed by the core test stage which included electronic eye tracking and data recording which provided information on the users' reading behaviour and on how frequently and how long individual design elements had been visited ${ }^{2}$ [10].

This part focused directly on the three websites selected for the user groups who were, first and foremost, asked whether they recognised the brand names in question and how often they had visited these websites previous to the survey. Furthermore, they were invited to rank the most common navigation tools (Table 1) according to their personal preferences and, subsequently, to rate the importance ${ }^{3}$ of the focal website features shown in Fig 4 . The students then tackled the most important part of the survey by indicating how they had personally experienced each site.

Table 1. Ranking of preferred navigation tools

\begin{tabular}{|l|c|c|c|c|}
\hline \multicolumn{1}{|c|}{ Preferred tools } & $\begin{array}{c}\text { Gemany } \\
(n=56)\end{array}$ & $\begin{array}{c}\text { Egypt } \\
(n=22)\end{array}$ & $\begin{array}{c}\text { China } \\
(n=28)\end{array}$ & $\begin{array}{c}\text { USA } \\
(n=64)\end{array}$ \\
\hline Navigation bars & 1 & 5 & 3 & 1 \\
\hline Hyperlinks in text & 2 & 4 & 4 & 3 \\
\hline $\begin{array}{l}\text { Graphics / } \\
\text { pictures }\end{array}$ & 3 & 2 & 1 & 2 \\
\hline $\begin{array}{l}\text { Search mask on } \\
\text { website }\end{array}$ & 4 & 6 & 2 & 4 \\
\hline Sitemaps & 5 & 7 & 5 & 5 \\
\hline Mouse-over links & 6 & 1 & 5 & 6 \\
\hline Pop-up windows & 7 & 3 & 3 & 7 \\
\hline No preference & 8 & 8 & 8 & 8 \\
\hline
\end{tabular}

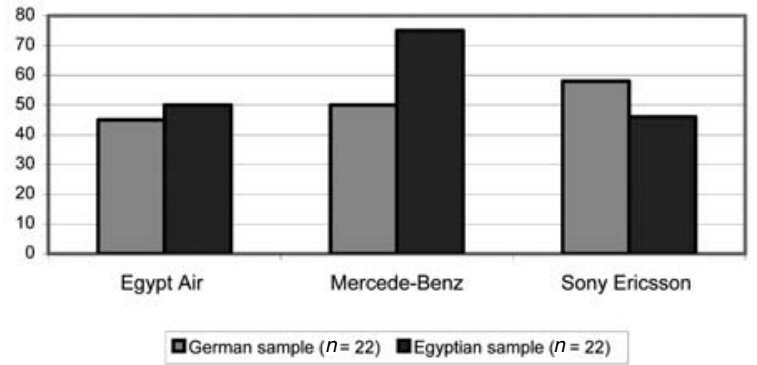

Fig 4. Overall appeal of the three websites

2 Each user was allowed 30 seconds per site. No introductory instructions were offered.

3 The rating scale included "highly important", "somewhat important" and "not important". "I do not know" was also admitted as an answer to enhance the results gained from this question. 
The post-test stage comprised the analysis of the data gathered, the preliminary review of the survey results and the discussion of the findings with the respondents.

\section{Analysing the data and interpreting the results}

\subsection{Tools and general web features}

The survey yielded significant data on reader behaviour and the user preferences of these relatively homogeneous multi-cultural samples.

All samples had preferred navigation tools which they rated as follows:

- The Chinese user group made first eye contact with the page centre, the Egyptian one with the top right, their US and German counterparts with the top left.

- All students welcomed supportive graphic elements which were especially appreciated by the Chinese.

- The German and US respondents used navigation bars and hyperlinks to guide them through their internet search.

- The Chinese sample looked, above all, for search masks in order to gain access to further details and instructions. The Egyptian students accessed specific information via mouse-over links and pulldown menus.

- Pop-ups were not popular with the majority of respondents - although about $50 \%$ of the Egyptian students did like those that offered genuine information instead of the unsolicited advertisements they often contain.

- Sitemaps were unpopular with most users with the exception of the German students who employed them more often than the other samples.

The ranking of the preferred website features (Table 2) revealed further noteworthy details:

- All users appreciated websites whose overall structure was easy on the eye and offered them a good overview of the information they expected to find.

- They all looked for adequate amounts of information. The Chinese sample desired a maximum amount of information whereas US and German users prefer sizeable amounts of information to be broken down into manageable core messages which are linked to further particulars or special offers.
Table 2. Overall ranking of the websites researched

\begin{tabular}{|l|c|c|c|c|c|c|}
\hline \multirow{2}{*}{$\begin{array}{c}\text { Preferred } \\
\text { website } \\
\text { features }\end{array}$} & \multicolumn{2}{|c|}{ Mercedes } & \multicolumn{2}{c|}{ Sony Ericsson } & \multicolumn{2}{c|}{ EgyptAir } \\
\cline { 2 - 7 }$(n=23)$ & $\begin{array}{c}\text { Germa- } \\
\text { ny } \\
(n=22)\end{array}$ & Egypt & $\begin{array}{c}\text { Germa- } \\
\text { ny }\end{array}$ & Egypt & $\begin{array}{c}\text { Germa- } \\
\text { ny }\end{array}$ \\
\hline $\begin{array}{l}\text { Attractively } \\
\text { designed }\end{array}$ & 1 & 2 & 2 & 3 & 3 & 1 \\
\hline $\begin{array}{l}\text { Harmonious } \\
\text { colours }\end{array}$ & 1 & 1 & 2 & 3 & 3 & 2 \\
\hline $\begin{array}{l}\text { Clearly } \\
\text { structured }\end{array}$ & 1 & 1 & 2 & 2 & 3 & 3 \\
\hline $\begin{array}{l}\text { Easily } \\
\text { understood }\end{array}$ & 2 & 1 & 2 & 3 & 1 & 2 \\
\hline $\begin{array}{l}\text { Informative } \\
\text { contents }\end{array}$ & 1 & 3 & 3 & 2 & 2 & 1 \\
\hline $\begin{array}{l}\text { State-of- } \\
\text { the-art }\end{array}$ & 1 & 2 & 2 & 1 & 3 & 3 \\
\hline $\begin{array}{l}\text { Overall } \\
\text { Ranking }\end{array}$ & $1^{\text {st }}$ & $1^{\text {st }}$ & $2^{\text {nd }}$ & $3^{\text {rd }}$ & $3^{\text {rd }}$ & $2^{\text {nd }}$ \\
\hline
\end{tabular}

- A good layout supported by illustrations and graphic design features was appreciated by all samples but no state-of-the-art version was required.

- Language and precise terminology were not conceived as essential assets as long as the message conveyed could be easily understood. Target-specific "in" language was judged positively by most respondents. For the Egyptian students appropriate terminology and language was more important than for the other samples.

- Colours did not play a major role either. Reassuring colours such as white and the lighter shades of blue were not particularly liked by the samples but generally accepted if they fitted the overall image of the website. More lively schemes would have been more successful with the young academics that contributed to this survey.

\subsection{Brand and market-specific analysis}

The following pages analyse the three websites produced for the Egyptian market. Samples 3 (8 female and 14 male Egyptian students) and 4 (16 female and 6 male German students) who were involved in this part of the survey provided a more comprehensive series of data which comprised, inter alia, the users' purchasing behaviour and their ownership of the consumer goods investigated.

It was found that $77 \%$ of the German and $68 \%$ of the Egyptian respondents originated from middle-class or professional family backgrounds sometimes with double incomes (36\% of the Egyptian mothers are housewives compared to $27 \%$ of the German ones). 
The students first stated whether they found the three websites appealing and then rated their individual features.

\subsubsection{EgyptAir}

EgyptAir is a national carrier which provides the same website for its domestic and its international customers. No translation into Arabic is available. The layout is dominated by a distinctive colour scheme that divides it into two major parts (Fig 5). Standard airline services are listed inconspicuously on the left for those users that are genuinely interested in purchasing the regular services, whereas topical news important for flyers from and to the Middle East, related EgyptAir services and special offers can be found on the right hand side at which Arab customers look first. This part is presented in the lighter and brighter colours preferred by over $50 \%$ of the Egyptian sample.

This website was not an unqualified success with any of the samples. Although brand recognition on the part of the Egyptian students was very high (90\% had used EgyptAir services to date), only $40 \%$ had visited the company's website and a mere $50 \%$ really favoured it. The majority felt that it was informative enough but looked too plain and lacked structure. Some of them missed a version in Arabic, especially as Egypt Air is a national flagship.

By contrast, $45 \%$ of the German sample found the website quite attractive (blue was a popular colour with this sample) and appreciated the information it offered on the EgyptAir itself and on its services. Only

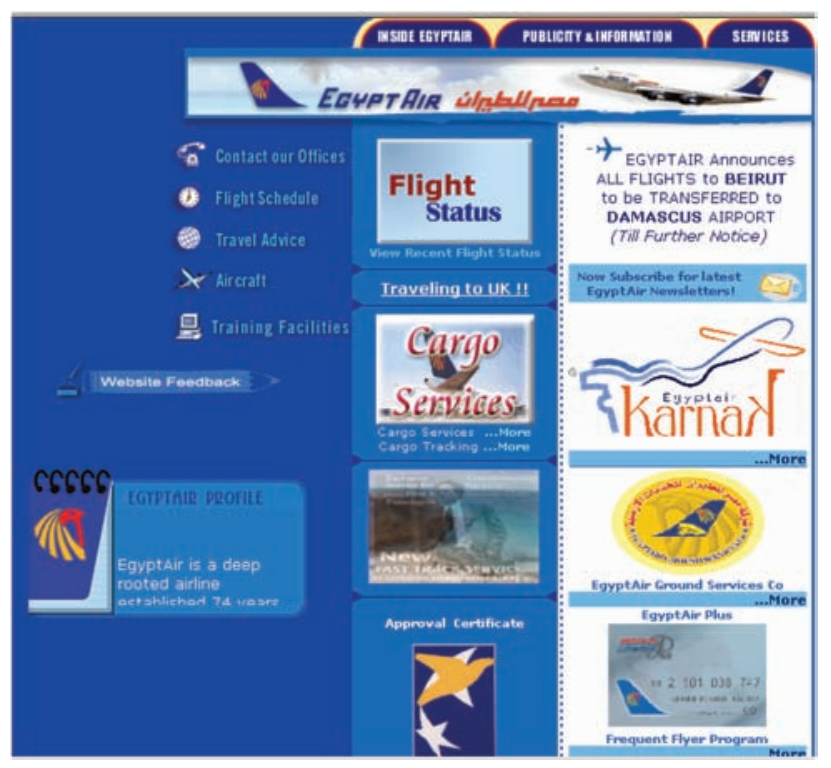

Fig 5. EgyptAir's website: Colour and symbols
$12 \%$ had looked at the carrier's online presentation before but, not surprisingly, over $50 \%$ were familiar with its name.

The specific features remembered best were the logo and the special offers (Table 3). The logo's exact position on the left and at the top left, however, was unfavourable from the perspective of the Egyptian users.

Table 3. Specific website features remembered

\begin{tabular}{|l|c|c|}
\hline \multicolumn{1}{|c|}{$n=22$} & Sample 3(\%) & Sample 4 (\%) \\
\hline Logo & 87 & 88 \\
\hline Exact position & 30 & 83 \\
\hline Special offers & 76 & 82 \\
\hline Instructive links & 61 & 47 \\
\hline $\begin{array}{l}\text { Appealing } \\
\text { illustrations }\end{array}$ & 51 & 43 \\
\hline Topical information & 53 & 28 \\
\hline
\end{tabular}

Although Egyptian students claim that "comfort" plays the most prominent role when they choose an airline (Fig 6), their high level of interest in special offers shows that they do have financial concerns but tend to express them differently.

In a cultural environment where financial status is a highly desirable social asset, it is easier to praise good offers than inexpensive services. The German students, on the other hand, openly state that they seek the most advantageous price in this complex market.

The charts indicate that EgyptAir might be well advised to review its websites for their young international customers. This site should mention its good track record in terms of reliability and safety and its consistent value for money policy. A second website should be created which is fully or partially in Arabic and places the company's logo in a more propitious position for its Arabic customers.

\subsubsection{Mercedes-Benz}

Egyptian car owners do not immediately associate the Mercedes-Benz brand with DaimlerChrysler and the

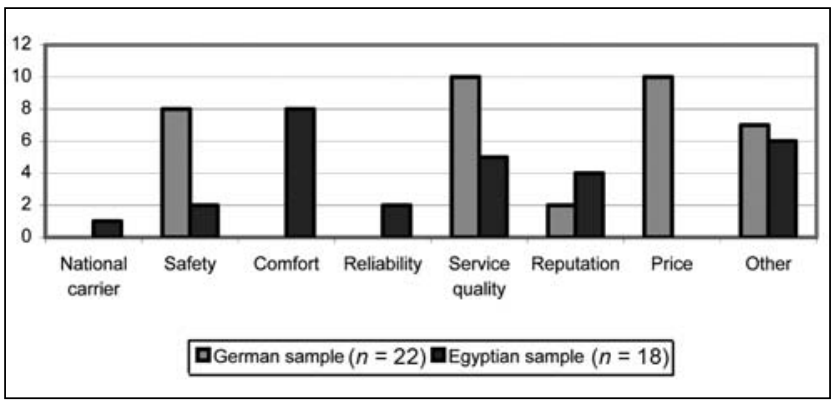

Fig 6. Reasons for choosing an airline 


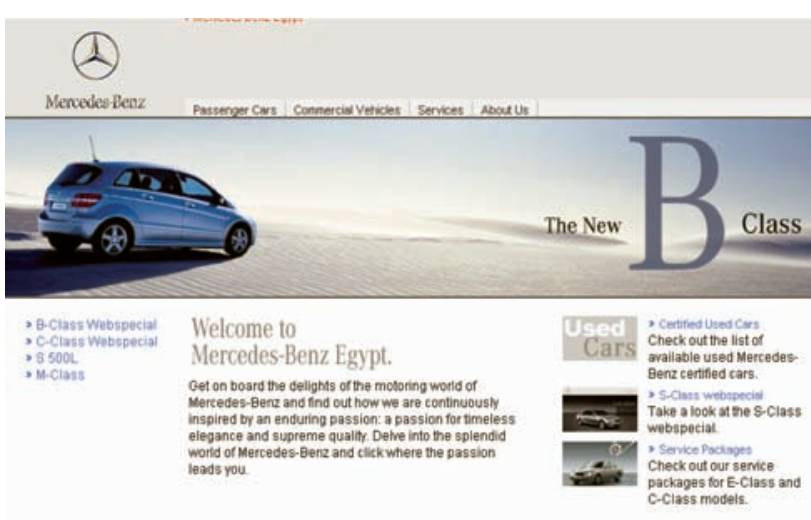

Fig 7. Mercedes-Benz Egypt: Selling a passionate experience

company therefore provides information on its different models rather than on itself ${ }^{4}$. The disproportionately tall letter B which introduces the new model on the right hand side of the Egyptian website is the eye-catcher that gives the product an identity (Fig 7). The communication policy underlying this site likens the experience of driving a $B$ type to a "passionate" and "delightful" event which constitutes a marked contrast to the quiet and light colours of the background.

The Mercedes-Benz brand is well-known in Egypt (sample 3-100\%; sample 4-97\%) and $56 \%$ of the Egyptian sample had visited the company's website before. $75 \%$ of the Egyptian students put it in first place whereas only half of the German sample thought it was the best of the three.

The Egyptian respondents (66\%) who own a car have purchased it on the grounds of price, user friendliness and quality. The same applies to the German sample (50\% are car owners) who additionally look for a smart design. The image of the car plays no particular role for these target groups (Fig 8).

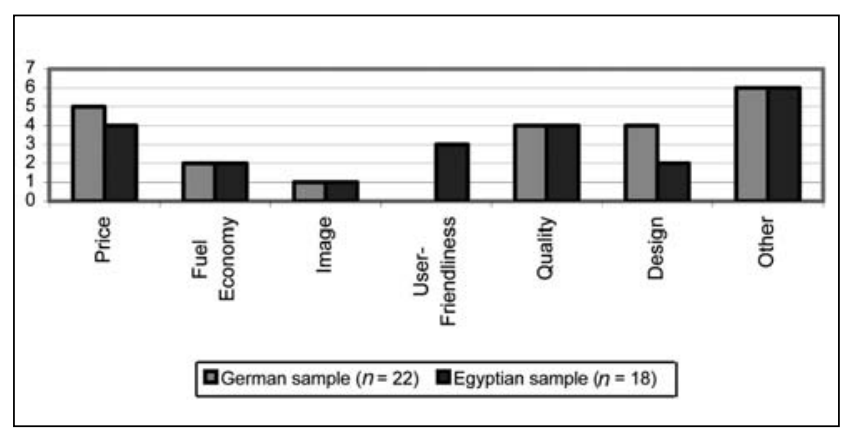

Fig 8. Reasons for choosing a car
Table 4. Specific features remembered

\begin{tabular}{|l|c|c|}
\hline \multicolumn{1}{|c|}{$n=22$} & Sample 3 (\%) & Sample 4 (\%) \\
\hline Logo & 100 & 97 \\
\hline Exact position & 40 & 84 \\
\hline $\begin{array}{l}\text { Appealing } \\
\text { illustrations }\end{array}$ & 82 & 73 \\
\hline Instructive links & 61 & 66 \\
\hline Topical information & 52 & 27 \\
\hline
\end{tabular}

The major difference in assessing the websites lay in the interpretation of the information provided (Table 4). The German sample rejected the emotional appeal of the script as uninformative. They also noticed that there was no information on the parent company with which they are familiar as two major DaimlerChrysler sites are located in the vicinity of their institution. The Egyptian students, on the other hand, accepted the message and the presentation of the model as genuine information. Although, most of them liked the elegant design, some thought it was a little too boring for their own market segment.

Both samples remembered seeing the Mercedes-Benz symbol but its position on the right was evidently not recorded by all Egyptian users.

The Egyptian students who assessed the site might buy this car for the comfort and quality it offers but they would be deterred by its price. This corresponds with the fact that Mercedes-Benz offer a second-hand service in Egypt.

On the whole, a more target-group oriented approach (e.g. less impressionistic colour schemes and a more dynamic environment) would have been welcomed by the two samples. In addition, the company might like to reconsider the position of its logo for the Egyptian market.

\subsubsection{Sony Ericsson}

This corporation's website emphasizes choice, a fresh start and holiday fun to its users and also promotes its products by means of prize competitions (Fig 9).

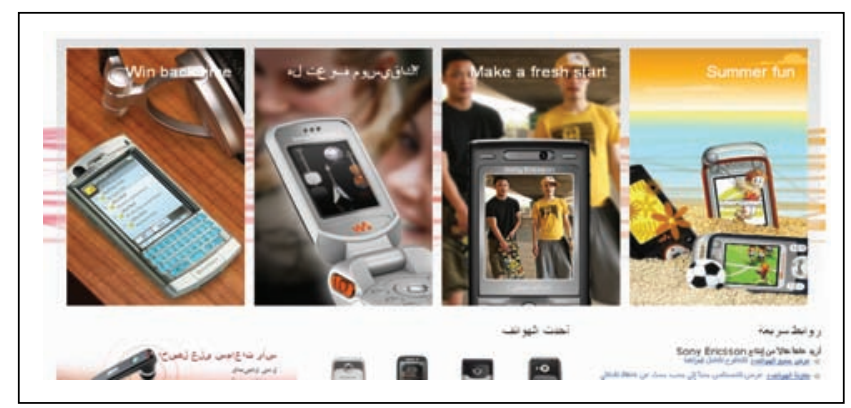

Fig 9. Sony Ericsson Egypt: Variety and fun 
Sony Ericsson is not a popular mobile phone brand in Egypt and none of the Egyptian respondents owned one. $82 \%$ use Nokia, a trusted brand which stands for quality. In the German sample, $41 \%$ had Nokia mobile phone; Sony Ericsson, Siemens, Samsung and Motorola products were also used to a much lesser extent.

It came as no surprise that all members of the German sample (vs. $66 \%$ of sample 3 ) were familiar with this company which is often quoted in their business lectures. In spite of this, the website visits of both samples were infrequent $(21 \%$ and $17 \%)$.

In line with the low brand recognition, less than half of the Egyptian sample found the Sony Ericsson website attractive, the most appealing feature being the product photos which focus on variety and choice, characteristics that are very important to some purchasers (Fig 10).

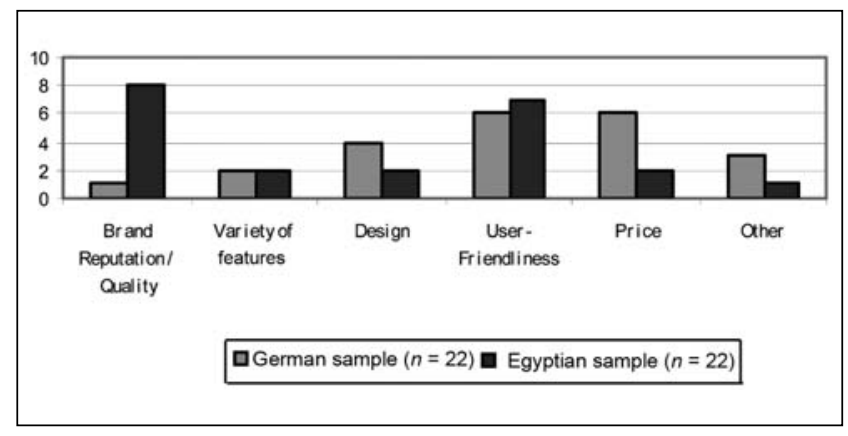

Fig 10. Reasons for purchasing a mobile phone

$58 \%$ of the German sample preferred this site to the others for its topicality (state-of-the-art models) and its links to useful additional information but were critical of the naturalistic pictorial overload and missed good special offers. Some of the Egyptian respondents felt that it was crowded and the information presented was scattered. The logo position on the right is unusual for German users and therefore hard to memorize (Table 5).

Table 5. Special features remembered

\begin{tabular}{|l|c|c|}
\hline \multicolumn{1}{|c|}{$n=22$} & Sample 3 (\%) & Sample 4 (\%) \\
\hline Logo & 76 & 85 \\
\hline Exact position & 50 & 15 \\
\hline $\begin{array}{l}\text { Appealing } \\
\text { illustrations }\end{array}$ & 81 & 20 \\
\hline Instructive links & 66 & 82 \\
\hline Topical information & 57 & 91 \\
\hline Special offers & 42 & 35 \\
\hline
\end{tabular}

The Sony Ericsson example shows that positive website assessment does not directly translate into purchasing behaviour. Mobile phone producers need to build up a positive image in their international markets before they can reach their targeted customers.

\section{Conclusions}

As the survey suggests that even in comparable target groups user behaviour coincides or differs subject to certain cultural aspects, international marketers should review their standardised communication policies for some markets and maintain them in others. The following guidelines might prove useful when making such a choice:

1) Target-market focused online communication needs to include cultural aspects such as reading behaviour at its most initial stage, i.e. its design. Reading behaviour, for instance, may encourage or prevent the memorisation of corporate logos.

2) For services (airlines) and products (mobile phones) that compete with local ones in markets with high cultural entrance barriers, translations into the local languages are advisable. Blended approaches such as the one adopted by Sony Ericsson represent a reasonable compromise.

3) In culturally related markets and with suitable target groups (as described above) standardized global online presentations which bypass cultural dissonances are advantageous. A case in point is the German and the US market where cost-effective standardised communication policy might be the strategy of choice.

4) Standardized approaches should not be dominated by Western or European design principles but should be adjusted to their target markets. Moreover they need to take design variations into account which depend on the type of the target market, the communication goals and the sector of industry involved.

\section{Research outlook}

This survey has produced valuable preliminary results which in view of the sample sizes will need to be tested against larger samples in order to arrive at a more comprehensive picture. These samples will also include more diverse user groups and also focus on the countries of the Enlarged European Union and the Mediterranean regions as little first-hand information is available with regard to these areas. Different products and markets will be included as a further step.

The user preferences stated by the samples of this sur- 
vey will be fine tuned by collecting and analysing data on their second and third choices made with a view to their preferred tools and features.

Last but not least, the new samples will be involved to a larger extent in the interpretation of the results as this will reinforce the recommendations arrived at.

\section{References}

1. ELASHMAWI, F.; HARRIS, P. R. Multicultural management 2000. Essential Cultural Insights for Global Business success. Houston: Verlag, 1998.

2. LEWIS, R. D. Cultural Imperative: Global Trends in the 21st Century. Boston: Intercultural Press, 2002.

3. REDDING, G.; STENING, B. W. Cross-Cultural Management. Northampton: Cheltenham and Camberley, 2003.

4. SCHNEIDER, S. C.; BARSOUX, J. Managing Across Cultures. Harlow: Pearson Educational Press, 2002.

5. SODERBERG, A. M.; HOLDEN, N. Rethinking Cross Cultural Management in a Globalizing Business World. International Journal of Cross Cultural Management, 2002, Vol 2, No 1, p. 103-121

6. THEUNER, G. Erfolgsfaktoren user-orientierter Webseitengestaltung, HMD Praxis der Wirtschaftsinformatik, Electronic Business, 2000, No 215, p. 69-73.

7. AMBLER, T., WITZEL, M. Doing Business in China. New York: Routledge, 2000.

8. THEUNER, G.; STEINMETZ, S. Eye tracking evaluation of Chinese websites for the Chinese market. International Business and Economics Research Journal, 2004, Vol 3, No 5, p. 47-55.

9. WANG, Y. et al. China: Business Culture. London: Thorogood Publishing Ltd, 2006.

10. DUCHOWSKI, A. T. Eye Tracking Methodology, Theory and Practice. New York, Heidelberg: Springer Publications, 2003. 